Datenschutzaufsicht (BayLDA) durchgeführt wurde und für die in erster Linie mittelständische Unternehmen befragt wurden.

Kunden müssen bereits bei der Datenerhebung über die geplante Verwendung ihrer personenbezogenen Daten unterrichtet werden. Bei der Datenschutzstudie 2012 gab jedoch jedes fünfte Unternehmen an, noch keine definierten Vorgaben dafür zu haben, wie Kunden über den Umgang mit personenbezogenen Daten informiert werden. Auch die Einwilligung zum Erhalt von Werbung, sofern sie gesetzlich erforderlich ist, wird nicht immer in schriftlicher oder elektronischer Form eingeholt. Ein weiterer Aspekt des Datenschutzes ist, dass Widersprüche zur Einwilligung werbegebundener Datennutzung zentral dokumentiert werden. Wird ein Widerspruch nicht richtig erfasst, kann es passieren, dass Kunden weiterhin Werbung erhalten. Auch in diesem Bereich zeigt die Studie bei 20 Prozent der Betriebe noch Nachholbedarf auf.

\section{Der Einsatz von „Sozialen Netzwerken“ an Schulen}

Handreichung des Ministeriums für Kultus, Jugend und Sport Baden-Württemberg.

Zusammenfassung:

Aufgrund datenschutzrechtlicher Bestimmungen ist die Verwendung von Sozialen Netzwerken für die dienstliche Verarbeitung personenbezogener Daten generell verboten. Hierunter fällt jegliche dienstlichen Zwecken dienende Kommunikation zwischen Schülern und Lehrkräften sowie zwischen Lehrkräften untereinander, ferner das (Zwischen-) Speichern von personenbezogenen Daten jeder Art auf Sozialen Netzwerken.

Im Rahmen des Unterrichts dürfen Soziale Netzwerke jedoch dazu genutzt werden, um Funktionsweise, Vorteile, Nachteile, Risiken usw. pädagogisch aufzuarbeiten.

Die Nutzung von Sozialen Netzwerken ist heutzutage unter Schülerinnen und Schülern sehr weit verbreitet. Zu den Sozialen Netzwerken zählen Produkte wie Face-book, Google+, Studi VZ aber auch Twitter. Diese Plattformen bieten sicherlich eine ganze Reihe von neuen, einfachen Möglichkeiten des Informationsaustausches und der Zusammenarbeit - auch für Lehrerkräfte. Daher ist es nachvollziehbar, dass bei-nahe die gesamte Kommunikation von Jugendlichen über Soziale Netzwerke abgewickelt wird. So werden neben Kurznachrichten und Chatfunktionen diverse Informationen in Blogs, Foren oder Gruppen ausgetauscht. Und auch in der Lehrerschaft ist ein zunehmendes Interesse an der Nutzung Sozialer Netzwerke zu erkennen. Leider sind die korrespondierenden rechtlichen Aspekte bei der Nutzung von Sozialen Netzwerken jedoch sehr komplex, gleichzeitig müssen die Schulen als staatliche Einrichtungen die gesetzlichen Rahmenbedingungen einhalten, auch das Landesdatenschutzgesetz beachten. Dieses Dokument soll eine erste Orientierung für die Verwendung von Sozialen Netzwerken an Schulen bieten.

Generell ist die Verarbeitung von personenbezogenen Daten im Rahmen der schulischen Arbeit auf Sozialen Netzwerken von Anbietern unzulässig, soweit deren Server außerhalb des europäischen Wirtschaftsraumes betrieben wer-den, es sich um US-Amerikanische Unternehmen handelt oder ein Zugriff von außerhalb des europäischen Wirtschaftsraumes möglich ist. Der Grund dafür ist, dass die dortigen Datenschutzstandards nicht mit deutschen und europäischen Datenschutzstandards in Einklang stehen. Ferner sind die AGBs bzw. Nutzungsbedingungen nicht mit dem deutschen Datenschutzrecht zu vereinbaren.

Daher ist von der Nutzung von sozialen Netzwerken zu dienstlichen Kommunikationszwecken abzusehen, da diese den geltenden Standards des Landesdatenschutzgesetzes (LDSG) nicht genügen. Dies bedeutet konkret für Lehrkräfte und Schulen, dass jegliche dienstliche Kommunikation auf oder mittels Sozialen Netzwerken sowohl zwischen Lehrkräften und Schülern als auch der Lehrkräfte untereinander unzulässig ist. Darunter fällt die Mailkommunikation innerhalb von Sozialen Netzwerken ebenso wie Chats, aber auch der dienstliche Austausch personenbezogener Daten wie das Mitteilen von Noten, ferner das Einrichten von Arbeitsund Lerngruppen zum Austausch von verschiedensten Materialien, die Vereinbarung schulischer Termine und Informationen zu Erziehungs- und Ordnungsmaßnahmen. Für alle diese Zwecke gibt es bereits Kommunikationswege, wie beispielsweise der konventionelle Schriftverkehr oder die Nutzung von verschlüsselten E-Mails einschlägiger Anbieter. Hinweise zur Nutzung von E-Mail finden Sie auf www.it.kultus-bw.de und dem Lehrerfortbildungsserver unter http://lehrerfortbildung-bw.de/sueb/recht/ds_neu/daten/email/

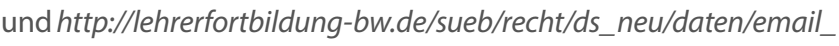
unter/. Ferner können Arbeits- und Lerngruppen oder der Austausch verschiedener Materialien beispielsweise mit Moodle (http://moodle.de/) als datenschutzfreundliche Alternative realisiert werden.

Zulässig ist jedoch die Behandlung von Sozialen Netzwerken im Unterricht. Dort können beispielsweise Funktionalitäten von Sozialen Netzwerken beleuchtet werden, deren Möglichkeiten und Risiken vorgestellt und diskutiert werden. Dies könnte auch dadurch erfolgen, dass auf freiwilliger Basis bereits vorhandene Accounts von Schülern genutzt werden. Dabei ist darauf zu achten, möglichst keine personenbezogenen Daten zu übermitteln. Nicht zulässig ist es jedoch, dass die Schüler im Unterricht dazu verpflichtet werden, in Sozialen Netzwerken Accounts anzulegen.

Obgleich auch die Nutzung von sogenannten Fanpages zur Selbstdarstellung von Schulen derzeit rechtlich umstritten ist, können solche Fanpages von Schulen genutzt werden. Das Kultusministerium empfiehlt jedoch, statt der Fanpages in Sozialen Netzwerken konventionelle Homepages zu realisieren und Fanpages nur als Zugang zur eigenen Internetseite der Schule zu nutzen. Auf keinen Falls dürfen personenbezogene Daten oder Bilder von Schülern oder anderen Personen auf Fanpages dargestellt werden. Nicht zulässig ist ferner, dass Schulen mit Schülern über diese Fanpages miteinander kommunizieren.

Das Installieren von sog. Social plug-ins, wie z.B. den „like-it“ Button von Facebook, auf Homepages von Schulen ist datenschutzrechtlich ebenfalls unzulässig.

Entscheidungsgrundlage für diese Entscheidung des Ministeriums für Kultus, Jugend und Sport ist unter anderem der Ergebnisbericht der Innenministerkonferenz vom 4. April 2012 in dem insbesondere festgestellt wird, dass derzeit die Sozialen Netzwerke zwar in vielen Bereichen Verbesserungen realisiert haben, jedoch nach wie vor datenschutzrechtlich unzulässig sind. Ferner der 30. Tätigkeitsbericht des Landesbeauftragten für den Datenschutz, in dem u.a. gefordert wird, von der Nutzung von Social plug-ins abzusehen, da diese den geltenden Datenschutzregelungen nicht genügen. 